\title{
EMERGÊNCIA E CRESCIMENTO INICIAL DE Tabebuia heptaphylla (Vell.) Toledo EM AMBIENTES CONTRASTANTES DE LUZ ${ }^{1}$
}

\begin{abstract}
Viviane Peixoto Borges², Maria Angélica Pereira de Carvalho Costa ${ }^{3}$ e Rogério Ferreira Ribas ${ }^{3}$
RESUMO - Tabebuia heptaphylla (Vell.) Toledo, popularmente conhecida como ipê-roxo ou ipê-roxo-desete-folhas, é uma Bignoniaceae nativa da Mata Atlântica, de importância econômica, medicinal e ornamental, além de ser indicada para reflorestamentos. Entretanto, informações ecofisiológicas sobre essa espécie ainda são escassas. Assim, visando dispor de informações que auxiliem na sua propagação e conservação, este trabalho avaliou a emergência e crescimento inicial dessa espécie em regimes contrastantes de luz. Foram realizados dois experimentos independentes. O primeiro em condições controladas, com os tratamentos $70 \%$ de sombreamento e a pleno sol; e o segundo em ambiente natural, com os tratamentos clareira e sub-bosque. Entre os parâmetros utilizados para avaliar a emergência sob influência das intensidades luminosas, apenas a porcentagem de emergência diferiu estatisticamente no ambiente natural, sendo superior na clareira. Em condições controladas, houve significância para a velocidade de emergência, sendo maior nas plantas submetidas ao a pleno sol. A espécie apresentou reduzido crescimento inicial no sub-bosque, sendo todos os parâmetros estatisticamente inferiores em relação às plantas da clareira, com exceção da razão de área foliar e área foliar específica. Em condições controladas, os resultados que diferiram significativamente foram todos superiores nas plantas a pleno sol. O estudo evidenciou que a espécie apresenta capacidade de formar plântulas em diferentes regimes de luz, inclusive sob forte sombreamento natural, porém essa condição reduz significativamente seu crescimento inicial, sendo este favorecido por maiores intensidades luminosas.
\end{abstract}

Palavras-chave: Ipê-roxo-de-sete-folhas; Recomposição florestal; Biomassa.

\section{EMERGENCY AND INITIAL GROWTH OF Tabebuia heptaphylla (Vell.) Toledo IN CONTRASTING LIGHT ENVIRONMENTS}

\begin{abstract}
Tabebuia heptaphylla (Vell.) Toledo, known as “ipê-roxo" or" ipê-roxo-de sete-folhas", is a native species of Atlantic Forest, with an economic, medicinal and ornamental role, suitable for reforestation. However, ecophysiological information about it is scarce. In order to provide information about propagation and conservation for this specie, the present study evaluated emergence and early growth of this species in different light exposure. We lead two independent trials. In the first we considered controlled conditions, with two treatments: $70 \%$ shade and full sun. The second was developed in a natural environment, with two treatments: gap and understory environments. From the total parameters used to evaluate the emergence of the species under influence of light intensities, only the emergence percentage was statistically different from the natural environment, with high values observed at gap treatment. Significant differences were observed in controlled environment, considering the emergence speed. This variable was higher in plants grown under full sun. Slower initial growth was observed for plants regenerated in the understory, compared to the plants growing in gaps, except for following characteristics: leaf area ration and specific leaf area. In controlled conditions, the results
\end{abstract}

\footnotetext{
${ }^{1}$ Recebido em 26.04.2012 aceito para publicação em 29.04.2014.

${ }^{2}$ Programa de Pós-Graduação em Ciências Agrárias, Universidade Federal do Recôncavo da Bahia, Bahia, Brasil. E-mail: <vivipborges@yahoo.com.br>.

${ }^{3}$ Centro de Ciências Agrárias, Biológicas e Ambientais, Universidade Federal de Recôncavo da Bahia, Bahia, Brasil. E-mail: <mapcosta63@gmail.com>e <ecofisiologia@gmail.com>.
} 
with higher significant differences were observed for plants under full sun. The study shows that the species has the capacity to germinate in different light conditions, even under strong natural shading. However, this condition reduces significantly its initial growth, which is favored by higher light intensities.

Keywords: Ipê-roxo-de-sete-folhas; Reforestation; Biomass.

\section{INTRODUÇÃO}

O ipê-roxo, ou ipê-roxo-de-sete-folhas (Tabebuia heptaphylla), é uma Bignoniaceae de grande porte que apresenta ampla dispersão no território brasileiro. Ocorre naturalmente da Bahia ao Rio Grande do Sul, tanto em florestas secundárias e primárias quanto em bordas de clareiras e clareiras pequenas. A espécie é muito explorada comercialmente devido à madeira de alta densidade, com grande resistência e maleabilidade, amplamente empregada na construção civil. Comumente utilizado no paisagismo urbano pela beleza da floração, o ipê-roxo-de-sete-folhas também apresenta propriedades medicinais na casca e nas folhas, com ação antiinflamatória, analgésica, diurética, inclusive anticancerígena (FALKENBERG, 1999; LORENZI, 2002; PARK et al., 2003; HIGA, 2006). Contudo, nas últimas décadas vem ocorrendo redução preocupante no número de exemplares em áreas de ocorrência natural, devido à ampla possibilidade de aproveitamento dessa espécie, somando-se à carência de informações sobre técnicas eficientes de cultivo (ETTORI, 1996; MARTO, 2005).

A propagação de grande número de espécies florestais encontra sérias limitações em razão do pouco conhecimento que se dispõe sobre suas características ecofisiológicas. Inúmeros fatores ambientais influenciam no desenvolvimento das espécies vegetais, e o suprimento inadequado de um desses fatores pode reduzir drasticamente o vigor da planta (FELFILI, 1999; SCALON et al., 2001). Entre esses fatores, a radiação luminosa é um dos mais importantes, pois exerce forte influência na taxa fotossintética, crescimento e arquitetura vegetal e pode apresentar efeitos sobre a germinação.

O processo de germinação é influenciado pela relação entre os comprimentos de onda vermelho e vermelhoextremo (620 a 775 nm). Essa relação a céu aberto está em torno de 1,2, enquanto no sub-bosque é em torno de 0,5(VAZQUEZ-YANES; OROZCO-SEGOVIA, 1993; MUNIZ, 2004). Em espécies pioneiras, o aumento dessa relação promove a germinação, enquanto espécies secundárias e clímax são capazes de se estabelecerem tanto sob os dosséis florestais quanto em clareiras.
Todas as plantas têm capacidade de modificar o seu modelo de desenvolvimento em resposta ao ambiente luminoso (HOLT, 1995). Porém, a natureza dessa resposta varia entre as espécies, de acordo com sua capacidade de aclimatação e a quantidade ou qualidade de luz, podendo classificá-las, de forma ampla, em dois grupos, plantas de sol ou de sombra (WALTERS; FIELD, 1987; SEEMANN, 1992; GRONINGER et al., 1996; LIMA et al., 2008).

A capacidade de uma espécie adaptar-se às condições de sol ou sombra pode ser evidenciada pela avaliação do crescimento inicial das plantas sob diferentes disponibilidades de luz (GONÇALVES et al., 2005; MIELKE; SCHAFFER, 2010). Experimentos realizados em condições controladas, onde a intensidade de radiação é previamente definida com o uso de sombreamento artificial, permitem verificar a tolerância das espécies à luz.

Tais estudos, entretanto, não refletem integralmente as condições proporcionadas pelo ambiente natural. Em um ambiente de clareira, a variação da irradiância depende, entre outros fatores, da posição do sol, da altura das árvores que estão ao redor e da dinâmica de passagem das nuvens, apresentando uma faixa de variação que vai de 25 a 2.300 ìmol fótons.m- ${ }^{2}$.s- ${ }^{1}$ ao longo de um único dia, enquanto a irradiância em um sub-bosque é predominantemente baixa e difusa, com pontuações de faixas de luz através das copas. Assim, estudos realizados em condições naturais fornecem dados mais consistentes sobre a tolerância das espécies florestais ao sombreamento e a sua sucessão ecológica (CHAZDON et al., 1996; BLOOR, 2003; GONÇALVES et al., 2005; LIMA et al., 2010).

Apesar do potencial do ipê-roxo, ainda são escassos estudos sobre sua plasticidade fisiológica durante a emergência e crescimento inicial. Tais estudos fornecem informações essenciais sobre as melhores condições de reprodução e propagação. Em geral, as pesquisas realizadas com a espécie abordam aspectos sobre os constituintes químicos (GARCEZ et al., 2007; MAHMOUD et al., 2011), qualidade fisiológica e 
conservação de sementes (AMARAL et al., 2011; MARTINS et al., 2012) e germinação e crescimento sob diferentes substratos (BOCHESE et al., 2008; SABONARO et al., 2009). Dessa forma, este trabalho objetivou avaliar o efeito de distintos regimes de luz sobre a emergência e crescimento inicial de Tabebuia heptaphylla em condições naturais e controladas.

\section{MATERIAL E MÉTODOS}

O estudo foi realizado no Município de Cruz das Almas, BA, situado no Recôncavo Baiano, a 1240’19”' de latitude Sul e $39^{\circ} 06^{\prime} 22^{\prime}$ ' de longitude Oeste e a 220 $m$ de altitude. O clima é tropical quente e úmido, Aw a Am, segundo a classificação de Köeppen, com temperatura média anual de $24,5^{\circ} \mathrm{C}$, umidade relativa de $80 \%$ e precipitação média de $1.249,7 \mathrm{~mm}$ anuais (AGRITEMPO, 2008).

Dois experimentos independentes foram realizados entre os meses de outubro de 2010 a fevereiro de 2011. O primeiro, em condições controladas, foi realizado no Campus do Centro de Ciências Agrárias, Ambientais e Biológicas da Universidade Federal do Recôncavo da Bahia (UFRB) e constituído dos tratamentos a pleno sol e a $70 \%$ de sombreamento, obtido com o uso de tela do tipo sombrite.

O segundo experimento, em condições naturais, foi realizado em um fragmento de Floresta de Transição Tropical Subperenifólia/Subcaducifólia, com os tratamentos sub-bosque, imposto pela cobertura vegetal, e clareira, cujo fluxo de radiação solar direta em nível do solo era, em média, de $4 \mathrm{~h}$ por dia. Estimativas de radiação luminosa, obtidas a partir da análise da cobertura vegetal por meio de fotos hemisféricas processadas pelo Programa Gap Light Analyzer-GLA (FRAZER et al., 1999), variaram de $23,18 \mathrm{~mol} \mathrm{~m}^{-2} \mathrm{~d}^{-1}$ na clareira e $3,83 \mathrm{~mol} \mathrm{~m}^{-2} \mathrm{~d}^{-1}$ no sub-bosque.

Para avaliação da emergência, sementes de $T$. heptaphylla, adquiridas da Flora Tietê Associação de Recuperação Florestal, foram acondicionadas em bandejas plásticas de 6,3 x 29,0 x 37,0 cm com areia lavada, nos locais de estudo. O delineamento experimental foi inteiramente casualizado e em cada tratamento, avaliadas cinco repetições com 40 sementes cada, em que cada repetição correspondeu a uma bandeja.

A emergência da plântula foi avaliada em dias alternados durante 40 dias. As variáveis calculadas foram: porcentagem de emergência $(\% E=N / A x 100$, em que $\mathrm{N}=\mathrm{n}^{\circ}$ de sementes germinadas; $\mathrm{A}=\mathrm{n}^{\circ}$ total de sementes); e índice de velocidade de emergência $(\mathrm{IVE}=\mathrm{N} 1 / \mathrm{T} 1+\mathrm{N} 2 / \mathrm{T} 2+\ldots+\mathrm{Nn} / \mathrm{Tn}$, em que $\mathrm{N}=$ número de sementes germinadas na primeira contagem, na segunda e na última contagem; $\mathrm{T}=\mathrm{n}^{\circ}$ de dias de semeadura da primeira, segunda e última contagens); tempo médio de emergência (TME = N1T1 + N2T2... + NnTn/ N1 + N2... + Nn, em que N1, N2 e Nn = números de sementes germinadas nos tempos T1, T2 e Tn, respectivamente) e velocidade média de emergência (VME: $1 / t$, em que $\mathrm{t}$ = tempo médio de emergência), determinados conforme Maguire (1962), Edmond e Drapala (1965) e Laboriau (1983).

Para avaliação do crescimento inicial, 40 plântulas por tratamento, apresentando cotilédones e ao menos um par de folhas primárias, foram transplantadas para vasos plásticos com volume de 1,7 L e mantidas nos mesmos tratamentos. O delineamento experimental foi do tipo inteiramente casualizado. No estudo em ambiente natural, foi utilizado o solo do próprio local como substrato, retirado da profundidade de 0 a 20 cm e homogeneizado. Em condições controladas, utilizou-se o substrato comercial Vivatto (Technes Agrícola Ltda.).

No transplante, 15 plântulas/tratamento foram avaliadas quanto a altura, número de folhas, diâmetro do colo (com o auxílio de paquímetro digital), massa seca de folhas (MSF), massa seca de caule (MSC), massa seca de raiz (MSR) e área foliar (AF). A massa seca foi obtida após a permanência do material vegetal em estufa de ventilação forçada a $\pm 70^{\circ} \mathrm{C}$ até atingir peso constante e posterior pesagem em balança analítica com precisão de 0,0001 g.

Ao final do experimento (95 dias na Mata e 85 dias na UFRB), esses parâmetros foram novamente quantificados e a partir desses dados, calculados em 10 plantas por tratamento: área foliar específica (AFE = AF/MSF); razão de área foliar (RAF = AF/MST); taxa de crescimento relativo $($ TCR $=(\operatorname{lnMST} 2-\operatorname{lnMST} 1)$ / (T2 - T1)); e taxa assimilatória líquida (TAL = [( MST2 - MST1)x(ln AF2 - ln AF1)/(AF2 - AF1)x(T2 - T1)]), por meio das fórmulas descritas por Briggs et al. (1920) e Hunt (1982).

Os resultados foram submetidos à análise de variância e teste F a 5\% de probabilidade, utilizando-se o Programa Statistica 7.0, para comparação das médias.

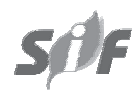

Revista Árvore, Viçosa-MG, v.38, n.3, p.523-531, 2014 


\section{RESULTADOS}

Em ambiente natural, a emergência das plântulas foi observada a partir do sétimo dia e oitavo dia após a semeadura, respectivamente no sub-bosque e na clareira. A porcentagem de emergência diferiu significativamente entre os tratamentos, tendo ocorrido maior formação de plântulas na clareira (Tabela 1).

Não ocorreram diferenças significativas entre os dois tratamentos quanto aos parâmetros relacionados à velocidade de emergência: índice de velocidade de emergência (IVE), tempo médio de emergência (TME) e velocidade média de emergência (VME).

Em condições controladas, as sementes submetidas a $70 \%$ de sombreamento e a pleno sol apresentaram porcentagem de emergência semelhante (Tabela 2). A emergência iniciou no sétimo dia após a semeadura a pleno sol e no sexto dia sob $70 \%$ de sombreamento.

Apenas o índice de velocidade de emergência diferiu significativamente entre os tratamentos, sendo superior nas plantas sombreadas.

O crescimento inicial das plântulas em condições naturais evidenciou grande variação na resposta da espécie aos níveis de radiação dos ambientes clareira e sub-bosque. Os valores médios do número de folhas,

Tabela 1 - Valores médios ( \pm erro-padrão) da porcentagem de emergência (\% emergência), índice de velocidade de emergência (IVE), tempo médio de emergência (TME) e velocidade média de emergência (VME) de sementes de Tabebuia heptaphylla aos 40 dias após a semeadura, em ambiente natural de clareira e sub-bosque na Mata da Cazuzinha, Cruz das Almas, BA.

Table 1 - Mean values ( \pm standard error) of emergence percentage (\% E), emergence speed index (IVE), emergence average time (TME) and emergence average speed (VME) of Tabebuia heptaphylla seeds 40 days after seeding in natural environment of gap and understory in Mata da Cazuzinha, Cruz das Almas, BA.

\begin{tabular}{lcc}
\hline Parâmetros & \multicolumn{2}{c}{ Tratamento } \\
\cline { 2 - 3 } & $77,00 \pm 3,98$ a & $56,00 \pm 7,61$ b \\
\hline$\%$ E & $20,18 \pm 0,97$ a & $17,50 \pm 3,05$ a \\
IVE & $27,70 \pm 1,35$ a & $21,30 \pm 3,26$ a \\
TME (dias) & $0,04 \pm 0,00$ a & $0,05 \pm 0,01$ a \\
\hline
\end{tabular}

Médias seguidas pela mesma letra não diferem entre si a 5\% de probabilidade.

Means followed by the same letter do not differ among them at 5\% probability.

Revista Árvore, Viçosa-MG, v.38, n.3, p.523-531, 2014
Tabela 2 - Valores médios ( \pm erro-padrão) da porcentagem de emergência (\% emergência), índice de velocidade de emergência (IVE), tempo médio de emergência (TME) e velocidade média de emergência (VME) de sementes de Tabebuia heptaphylla aos 40 dias após a semeadura, em condições controladas sob $70 \%$ de sombreamento e a pleno sol, em Cruz das Almas, BA.

Table 2-Mean values ( \pm standard error) of emergence percentage (\% E), emergence speed index (IVE), emergence average time (TME) and emergence average speed (VME) of Tabebuia heptaphylla seeds 40 days after seeding, under controlled conditions in $70 \%$ shade and full sun, in Cruz das Almas, BA.

\begin{tabular}{|c|c|c|}
\hline \multirow[t]{2}{*}{ Parâmetros } & \multicolumn{2}{|c|}{ Tratamento } \\
\hline & Pleno sol & $\begin{array}{c}\text { Sombreamento } \\
70 \% \\
\end{array}$ \\
\hline$\% \mathrm{E}$ & $51,00 \pm 3,41 \mathrm{a}$ & $60,50 \pm 3,20$ \\
\hline IVE & $13,66 \pm 1,15 b$ & $18,21 \pm 1,08$ \\
\hline TME (dias) & $18,81 \pm 1,45$ a & $22,76 \pm 1,24 a$ \\
\hline VME (sementes.dia ${ }^{-1}$ ) & $0,05 \pm 0,00 \mathrm{a}$ & $0,04 \pm 0,00 a$ \\
\hline
\end{tabular}

Médias seguidas pela mesma letra não diferem entre si a 5\% de probabilidade.

Means followed by the same letter do not differ among them at 5\% probability.

altura, diâmetro de caule, partição de biomassa, área foliar, taxa de crescimento relativo e taxa assimilatória líquida foram todos significativamente maiores na clareira (Tabela 3).

Para a área foliar específica, que está relacionada com a espessura foliar, as plantas do sub-bosque apresentaram maior valor significativo. Nesse ambiente, também foi observada maior razão de área foliar.

Observou-se que em condições controladas os resultados foram menos contrastantes, tendo diferido entre os tratamentos apenas os parâmetros número de folhas, massa seca de folhas, massa seca total, taxa de crescimento relativo (TCR) e taxa assimilatória líquida (TAL) (Tabela 4).

\section{DISCUSSÃO}

A emergência nos dois locais de estudos iniciou entre o sexto e oitavo dia após o plantio, tempo menor que o indicado por Lorenzi (2002), o qual informa que a emergência dessa espécie ocorre de 10 a 12 dias.

Em ambiente natural, a menor porcentagem de emergência encontrada no sub-bosque demonstra que a menor disponibilidade de luz e, possivelmente, a qualidade dessa luz reduzem a capacidade de emergência 
Tabela 3 - Valores médios ( \pm erro-padrão) do número de folhas (NF), altura de planta (AP), diâmetro do caule (DC), massa seca de folhas (MSF), massa seca de caule (MSC), massa seca de raiz (MSR), massa seca total (MST), área foliar (AF), área foliar específica (AFE), razão de área foliar (RAF), taxa de crescimento relativo (TCR) e taxa assimilatória líquida (TAL) em plantas de Tabebuia heptaphylla aos 130 dias após a emergência, em ambiente natural de clareira e sub-bosque, na Mata da Cazuzinha, Cruz das Almas, BA.

Table 3 - Mean values ( \pm standard error) of leaf number (NF), plant height (AP), stem diameter (DC), leaf dry mass (MSF), stem dry mass (MSC), root dry mass (MSR), total dry mass (MST), leaf area (AF), specific leaf area (AFE), leaf area ratio (RAF), relative growth rate (TCR) and net assimilation rate (TAL) in plants of Tabebuia heptaphylla 130 days after emergence, in natural environment of gap and understory, in Mata da Cazuzinha, Cruz das Almas, BA.

\begin{tabular}{lcc}
\hline Parâmetros & \multicolumn{2}{c}{ Tratamento } \\
\cline { 2 - 3 } & Clareira & Sub-bosque \\
\hline NF & $15,00 \pm 1,38 \mathrm{a}$ & $9,00 \pm 0,37 \mathrm{~b}$ \\
AP $(\mathrm{cm})$ & $17,58 \pm 1,75 \mathrm{a}$ & $8,22 \pm 0,64 \mathrm{~b}$ \\
DC $(\mathrm{mm})$ & $3,77 \pm 0,23 \mathrm{a}$ & $1,96 \pm 0,02 \mathrm{~b}$ \\
MSF $(\mathrm{g})$ & $0,95 \pm 0,06 \mathrm{a}$ & $0,11 \pm 0,00 \mathrm{~b}$ \\
MSC (g) & $0,31 \pm 0,03 \mathrm{a}$ & $0,04 \pm 0,00 \mathrm{~b}$ \\
MSR $(\mathrm{g})$ & $0,67 \pm 0,08 \mathrm{a}$ & $0,07 \pm 0,01 \mathrm{~b}$ \\
MST $(\mathrm{g})$ & $1,93 \pm 0,14 \mathrm{a}$ & $0,22 \pm 0,01 \mathrm{~b}$ \\
AF $\left(\mathrm{cm}^{2}\right)$ & $226,67 \pm 15,05 \mathrm{a}$ & $41,60 \pm 2,04 \mathrm{~b}$ \\
AFE $\left(\mathrm{cm}^{2} \cdot \mathrm{g}^{-1}\right)$ & $240,15 \pm 5,32 \mathrm{~b}$ & $375,23 \pm 24,91 \mathrm{a}$ \\
RAF $\left(\mathrm{cg}^{-1} \mathrm{~m}^{-1}\right)$ & $118,09 \pm 2,98 \mathrm{~b}$ & $189,62 \pm 16,90 \mathrm{a}$ \\
TCR $\left(\mathrm{mg}^{-1} \cdot \mathrm{gdia}^{-1}\right)$ & $23,54 \pm 0,79 \mathrm{a}$ & $20,62 \pm 0,65 \mathrm{~b}$ \\
TAL $\left(\mathrm{cm}^{-2} \cdot \mathrm{dia}^{-1}\right)$ & $0,16 \pm 0,01 \mathrm{a}$ & $0,09 \pm 0,01 \mathrm{~b}$ \\
\hline
\end{tabular}

Médias seguidas pela mesma letra não diferem entre si a 5\% de probabilidade.

Means followed by the same letter do not differ among them at $5 \%$ probability.

dessa espécie. Entretanto, observa-se a capacidade de formar plântulas sob o dossel florestal, em ausência de luz solar direta, característica essa das espécies de estádios mais avançados de sucessão.

Em condições controladas, os valores encontrados foram próximos àqueles obtidos por Bochese et al. (2008), ao avaliarem a emergência de T. heptaphylla sob $70 \%$ de sombreamento e diferentes substratos: emergência a partir do sexto dia após a semeadura, com o maior percentual equivalente a 42,08\%. Resultado semelhante também foi obtido por Martins et al. (2012), que obtiveram taxa de $46 \%$ ao avaliar a emergência de $T$. heptaphylla em bandejas com areia sob luminosidade indireta.
Tabela 4 - Valores médios ( \pm erro-padrão) do número de folhas (NF), altura de planta (AP), diâmetro do caule (DC), massa seca de folhas (MSF), massa seca de caule (MSC), massa seca de raiz (MSR), massa seca total (MST), área foliar (AF), área foliar específica (AFE), razão de área foliar (RAF), taxa de crescimento relativo (TCR) e taxa assimilatória líquida (TAL) em plantas de Tabebuia heptaphylla aos 120 dias após a emergência, em ambiente controlado sob $70 \%$ de sombreamento e a pleno sol, em Cruz das Almas, BA.

Table 4-Mean values ( \pm standard error) of leaf number (NF), plant height (AP), stem diameter (DC), leaf dry mass (MSF), stem dry mass (MSC), root dry mass (MSR), total dry mass (MST), leaf area (AF), specific leaf area (AFE), leaf area ratio $(R A F)$, relative growth rate (TCR) and net assimilation rate (TAL) in plants of Tabebuia heptaphylla 120 days after emergence, under controlled conditions in $70 \%$ shade and full sun, in Cruz das Almas, BA.

\begin{tabular}{|c|c|c|}
\hline \multirow{2}{*}{ Parâmetros } & \multicolumn{2}{|c|}{ Tratamento } \\
\hline & Pleno sol & $\begin{array}{c}\text { Sombreamento } \\
70 \% \\
\end{array}$ \\
\hline $\mathrm{NF}$ & $18,60 \pm 0,67 a$ & $15,80 \pm 0,20 b$ \\
\hline AP $(\mathrm{cm})$ & $22,32 \pm 1,02 \mathrm{a}$ & $23,22 \pm 0,96 a$ \\
\hline DC $(r$ & $5,12 \pm 0,16$ a & $4,85 \pm 0,09 a$ \\
\hline MSF (g) & $3,10 \pm 0,18$ a & $2,23 \pm 0,14 \mathrm{~b}$ \\
\hline MSC (g) & $0,93 \pm 0,06 \mathrm{a}$ & $0,80 \pm 0,05 a$ \\
\hline MSR (g) & $2,79 \pm 0,24 \mathrm{a}$ & $2,38 \pm 0,22 a$ \\
\hline MST (g) & $6,82 \pm 0,34 a$ & $5,41 \pm 0,32 b$ \\
\hline $\mathrm{AF}\left(\mathrm{cm}^{2}\right)$ & $650,5 \pm 58,5 \mathrm{a}$ & $530,8 \pm 32,8$ a \\
\hline $\operatorname{AFE}\left(\mathrm{cm}^{2} \cdot \mathrm{g}^{-1}\right)$ & $210,0 \pm 5,32$ a & $239,1 \pm 5,82$ a \\
\hline RAF $\left(\mathrm{cg} \cdot \mathrm{m}^{-1}\right)$ & $95,58 \pm 7,79 a$ & $99,45 \pm 5,19 a$ \\
\hline TCR (mg.g ${ }^{-1} \cdot$ dia $\left.^{-1}\right)$ & $50,29 \pm 0,87 a$ & $44,41 \pm 0,95 b$ \\
\hline TAL $\left(\mathrm{cm}^{-2} \cdot \operatorname{dia}^{-1}\right)$ & $0,53 \pm 0,03 \mathrm{a}$ & $0,44 \pm 0,02 \mathrm{~b}$ \\
\hline
\end{tabular}

Médias seguidas pela mesma letra não diferem entre si a 5\% de probabilidade.

Means followed by the same letter do not differ among them at 5\% probability.

A não ocorrência de variação significativa para a porcentagem de emergência entre os tratamentos a pleno sol e a $70 \%$ de sombreamento permite verificar que essa espécie apresenta considerável plasticidade em relação ao processo germinativo. Tal fato indica que, havendo disponibilidade hídrica, suas sementes podem germinar e formar plântulas tanto em ambientes totalmente abertos quanto em locais sombreados. Aguiar et al. (2005) encontraram resultados semelhantes ao avaliarem a germinação de Caesalpinia echinata (pau-brasil) a pleno sol e em níveis de sombreamento, em que não foi observada variação significativa para a porcentagem e velocidade de emergência. Diferentemente, Fonseca 
et al. (2006), ao avaliarem a emergência de Pseudopiptadenia psilostachya, encontraram menor taxa de emergência nas plantas a pleno sol em relação àquelas a $70 \%$ de sombreamento.

Em relação ao crescimento inicial das plântulas, os resultados obtidos em condições naturais evidenciaram crescimento pouco expressivo sob o dossel florestal. Siebeneichler et al. (2008), ao estudarem o comportamento da mesma espécie em diferentes condições de luminosidade, também encontraram valores inferiores de altura, diâmetro e biomassa nas plantas submetidas ao sombreamento natural.

Em geral, há redução no crescimento conforme aumento do sombreamento devido à menor taxa fotossintética e, consequentemente, menor produção de fotoassimilados. Resultados semelhantes também foram observados em outras espécies (POPMA; BONGERS, 1988; COURT; MITCHELL, 1989; OSUNKOYA;ASH, 1991; MARIMON et al., 2008; SILVA et al., 2010). Lima et al. (2008), ao estudarem o comportamento de Caesalpinia ferrea em diferentes luminosidades, também encontraram inibição no crescimento inicial de mudas submetidas ao sombreamento natural.

Para a área foliar e massa seca total, as plantas da clareira apresentaram valores de $445 \%$ e $777,3 \%$ maiores que as do sub-bosque, respectivamente. Esses resultados comprovam a forte inibição de crescimento que as plantas sob sombreamento natural sofreram, devido às limitações fisiológicas impostas pela baixa luminosidade.

O maior valor de área foliar específica nas plantas do sub-bosque é explicado pelo fato de que, sob baixa luminosidade, as plantas apresentam folhas mais delgadas, pois possuem cutícula, epiderme e parênquimas menos espessos que aquelas submetidas a maior intensidade luminosa.

A taxa de crescimento relativo (TCR) é uma taxa de crescimento comparativa com o crescimento inicial e dependente de alteração na fisiologia, medida pela taxa assimilatória líquida (TAL) e, ou, na morfologia da planta, medida pela razão de área foliar (RAF) (HUNT, 1982). Por sua vez, a TAL é uma estimativa da fotossíntese líquida, e a RAF representa a relação entre a área foliar e a biomassa total da planta (PEIXOTO et al., 2011). Nas plantas crescidas na clareira, a maior TAL proporcionou maior TCR. Entretanto, observa-se que a RAF foi superior nas plantas do sub-bosque. Segundo Benincasa (2003), a RAF decresce conforme o aumento na disponibilidade de luz, pois, quanto maior a intensidade luminosa, menor a área foliar necessária para produzir massa seca.

Em condições controladas, os resultados superiores de massa seca de folhas e massa seca total nas plantas a pleno sol indicaram que nessa espécie, durante a fase inicial de crescimento, o acúmulo de biomassa é favorecido pela maior irradiância. Oliveira e Gualtieri (2011), estudando o crescimento inicial de Tabebuia aurea sob três níveis de luz, concluíram que essa espécie também apresenta maior acúmulo de biomassa quando cultivada em maior intensidade luminosa.

De acordo com Larcher (2004), as plantas que crescem sob forte radiação desenvolvem folhas espessas e apresentam metabolismo mais ativo e, como consequência, essas plantas apresentam maior produção de massa seca, com maior conteúdo energético.

A TCR foi significativamente maior no tratamento a pleno sol que no sombrite. Esse maior crescimento e, consequentemente, a produtividade biológica das plantas sob alta radiação ocorreram devido à maior TAL (Tabela 4). Batiston et al. (2008), avaliando o crescimento inicial de Machaerium sp., também encontraram maiores valores de TCR e TAL nas plantas a pleno sol, aos 120 DAS.

Os resultados encontrados neste estudo concordam com os obtidos por Siebeneichler et al. (2008), que verificaram crescimento semelhante de T. heptaphylla quando submetida a 50 e $100 \%$ de luminosidade.

\section{CONCLUSÕES}

A espécie Tabebuia heptaphylla possui capacidade de regeneração em ambientes ensolarados e sombreados.

As plantas apresentam crescimento lento no subbosque, contudo sobrevivem a essa condição de sombreamento.

As maiores taxas de crescimento e acúmulo de biomassa durante o crescimento inicial são encontradas nas plantas cultivadas a pleno sol, podendo ser realizada a produção de mudas nessa condição.

Revista Árvore, Viçosa-MG, v.38, n.3, p.523-531, 2014

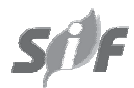




\section{AGRADECIMENTOS}

À CAPES, pela concessão da bolsa de Mestrado à primeira autora; e à Secretaria de Agricultura e Meio Ambiente do Município de Cruz das Almas, BA, pelo apoio durante a condução do experimento na Mata da Cazuzinha.

\section{REFERÊNCIAS}

AGRITEMPO. Disponível em: http:// www.agritempo.gov.br/agroclima/sumario. Acesso em: 10 de jul. 2011.

AGUIAR, F. F. A. et al. Germinação de sementes e formação de mudas de Caesalpinia echinata Lam. (Pau-Brasil): Efeito de sombreamento. Revista Árvore, v.29, n.6, p.871-875, 2005.

AMARAL, J. B. et al. Teste de raios X para avaliação do potencial fisiológico de sementes de ipê-roxo. Revista Brasileira de Sementes, v.33, n.4, p.601-607, 2011.

BATISTON, D. A.; ARANTES, W. M.; ALMEIDA, V. P. Crescimento inicial de Machaerium SP (Leguminosae-Papilonoideae) em resposta a diferentes condições de sombreamento. Revista Eletrônica de Biologia, v.1, n.1, p.8-20, 2008.

BENINCASA, M. M. P. Análise de crescimento de plantas (noções básicas). 2.ed Jaboticabal: FUNEP, 2003. 41p.

BLOOR, J. M. G. Light responses of shadetolerant tropical tree species in north-east Queensland: a comparison of forest- and shadehouse-grown seedlings. Journal of Tropical Ecology, v.19, n.1, p.163-170, 2003.

BOCCHESE, R. A. et al. Efeito de diferentes tipos de solos na germinação de sementes de Tabebuia heptaphylla, em casa telada. Cerne, v.14, n.1, p.62-67, 2008.

BRIGGS, G. E.; KIDD, E.; WEST, C. A.

Quantitative analysis of plant growth. Annals of Applied Biology, v.7, n.1, p.103-23, 1920.

CHAZDON, R. L. et al. Photosynthetic responses of tropical forest plants to contrasting light environments. In: MULKEY, S. S.; CHAZDON, R. L.; SMITH, A. P. (Ed.) Tropical forest plant ecophysiology. New York: Chapman and Hall, 1996. p.5-55.
COURT, A. J.; MITCHELL, N. D. The growth responses of Dysoxylum spectabile (Meliaceae) to a shaded environment. New Zealand Journal of Botany, v.27, n.3,p. 353-358, 1989.

EDMOND, J. B.; DRAPALA, W. J. The effects of temperature, sand and soil, and acetone on germination of okra seed. Proceedings of the American Journal Society for Horticultural Science, v.71, p.428-434, 1965.

ETTORI, L. C. et al. Variabilidade genética em populações de Ipê-roxo - Tabebuia heptaphylla (Vell.) Tol. - para conservação ex situ. Revista do Instituto Florestal, v.8, n.1, p.61-70, 1996.

FALKENBERG, M. B. Quinonas. In: SIMÕES, C. M. O. et al. (Org.). Farmacognosia - da planta ao medicamento. Porto Alegre: UFRGS/UFSC, 1999. 821p.

FELFILI, J. M. et al. Comportamento de plântulas de Sclerolobium paniculatum Vog. var. rubiginosum (Tul.) Benth. sob diferentes níveis de sombreamento, em viveiro. Revista Brasileira de Botânica, v.22, n.2 (suplemento), p.297-301, 1999.

FONSECA, M. G.; LEÃO, N. V. M.; SANTOS, F. A. M. Germinação e crescimento inicial de plântulas de Pseudopiptadenia psilostachya (DC.) G.P. Lewis \& M.P. Lima (Leguminosae) em diferentes ambientes de luz. Revista Árvore, v.30, n.6, p.885-891, 2006.

FRAZER, G. W.; CANHAM, C. D.; LERTZMAN, K. P. Gap Light Analyzer (GLA), Version 2.0: Imaging software to extract canopy structure and gap light transmission indices from truecolour fisheye photographs, users manual and program documentation. Copyright (C) 1999.

GARCEZ, F. R. et al. Novos constituintes químicas das cascas do caule de Tabebuia heptaphylla. Química Nova, v.30, p.18871891, 2007.

GONÇALVES, J. F. C. t al. Growth, photosynthesis and stress indicators in Young rose Wood plants (Aniba rosaeodora Ducke) under different light intensities. Brazilian Journal of Plant Physiology, v.17, n.3, p.325-334, 2005.

Revista Árvore, Viçosa-MG, v.38, n.3, p.523-531, 2014 
GRONINGER, J. W. et al. Growth and photosynthesis responses or four Virginia Piedmont tree species to shade. Tree Physiology, v.16, n.9, p.773-778, 1996.

HIGA, T. C. Morfogênese e Conservação in vitro para Tabebuia heptaphylla (Vellozo) Toledo (Bignoniaceae). 2006. 134f. Dissertação (Mestrado em Recursos Genéticos Vegetais) - Universidade Federal de Santa Catarina, Porto Alegre, 2006.

HOLT, J. S. Plant response to light: a potencial tool for weed management. Weed Science, v.43, p.474-482, 1995.

HUNT, R. Plant growth curves: the functional approach to plant growth analysis. London: Edward Arnold, 1982.

LABOURIAU, L. G. A germinação de sementes. Washington: OEA, 1983. 174 p.

LARCHER, W. Ecofisiologia vegetal. São Carlos: RiMA Artes e Textos, 2004. 531p.

LIMA, J. D. et al. Efeitos da luminosidade no crescimento de mudas de Caesalpinia ferrea Mart. ExTul. (Leguminosae, Caesalpinoideae). Acta Amazonica, v.38, n.1, p.5-10, 2008.

LIMA, M. A. O. et al. Crescimento e plasticidade fenotípica de três espécies arbóreas com uso potencial em sistemas agroflorestais. Scientia Forestalis, v.38, n.87, p.527-534, 2010.

LORENZI, H. Árvores brasileiras: manual de identificação e cultivo de plantas arbóreas do Brasil. 2.ed. Nova Odessa, Instituto Plantarum, 2002. v.2. 384p.

MAGUIRE, J. D. Speed of germination-aid selection evolution for sudling emergence and vigor. Crop Science, v.2, n.2, p.176-177, 1962.

MAHMOUD, T. S. et al. In vitro cytotoxic activity of Brazilian Middle West plant extracts.

Brazilian Journal of Pharmacognosy, v.21, n.3, p.456-464, 2011.

MARIMON, B. S. et al. Desenvolvimento inicial e partição de biomassa de Brosimum rubescens Taub. (Moraceae) sob diferentes níveis de sombreamento. Acta Botanica Brasilica, v.22, n.4, p.941-953, 2008.
MARTINS, L.; LAGO, A. A.; CÍCERO, S. M. Conservação de sementes de ipê-roxo. Revista Brasileira de Engenharia Agrícola e Ambiental, v.16, n.1, p.108-112, 2012.

MARTO, G. B. T. Identificação de espécies florestais: Tabebuila heptaphylla (Ipê-roxo). IPEF - Instituto de Pesquisa e Estudos Florestais, 2005. Disponível em <http://www.ipef.br/ identificacao/tabebuia.heptaphylla.asp> Acesso em: 25 de abril de 2013.

MIELKE, M. S.; SCHAFFER, B. Photosynthetic and growth responses of Eugenia uniflora L. seedlings to soil flooding and light intensity. Environmental and Experimental Botany, v.68, n.2, p.113-121, 2010.

MUNIZ, M. R. A. Estudo do regime de luz nas quatro principais formações fitogeográficas no Estado de São Paulo durante o inverno do ano de 2003. 2004 190f. Dissertação (Mestrado em Biologia Vegetal) Universidade Estadual de Campinas, Campinas, 2004.

OLIVEIRA, A. K. M.; GUALTIERE, S. C. J. Crescimento inicial de Tabebuia áurea sob três intensidades luminosas. REVSBAU, v.6, n.1, p.90-103, 2011

OSUNKOYA, O. O.; ASH, J. E. Acclimation to a change in light regime in seedlings of six Australian rainforest tree species. Australian Journal of Botany, v.39, n.6, p.591-605, 1991.

PARK, B. S. et al. Antioxidant activity and characterization of volatile constituents of Taheebo (Tabebuia impetiginosa Martius ex DC). Journal Agriculture Food Chemistry, v.51, n.1, p.295-300, 2003.

PEIXOTO, C. P.; CRUZ, T. V.; PEIXOTO, M. F. S. P. Análise quantitativa do crescimento de plantas: conceitos e prática. Enciclopédia Biosfera, v.7, n.1, p.51-76, 2011.

POPMA, J.; BONGERS, F. The effect of canopy gaps on growth and morphology of seedlings of rain forest species. Oecologia, v.75, n.4, p.625-632, 1988.

SABONARO, D. Z. et al. Producción de plantas de Tabebuia heptaphylla en diferentes sustratos y niveles de irrigación, en condiciones de invernadero. Bosque, v.30, n.1, p.27-35, 2009. 
SCALON, S. P. Q. et al. Germinação e crescimento de mudas de pitangueira (Eugenia uniflora L.) sob condições de sombreamento. Revista

Brasileira de Fruticultura, v.23, n.3, p.652-655, 2001.

SEEMANN, J. R. Light adaptation/acclimation of photosynthesis and the regulation of ribulose-1,5bisphosphate carboxylase activity in sun and shade plants. Plant Physiology, v.91, n.1, p.118, 1992.

SIEBENEICHLER, S. C. et al. Características morfosiológicas em plantas de Tabebuia heptaphylla (Vell.) Tol. em condições de luminosidade. Acta Amazônica, v.38, n.3, p.467-472, 2008.
SILVA, R. R. et al. Potencial de desenvolvimento inicial da Aspidosperma polyneuron Müll em projetos de recuperação de área degradada na região sudeste da Amazônia Legal. Revista Brasileira de Tecnologia Aplicada nas Ciências Agrárias, v.2, n.1, p.71-78, 2010.

VÁZQUEZ-YANES, C.; OROZCO-SEGOVIA, A. Patterns of seed longevity and germination in the tropical rainforest. Annual Review in Ecology and Systematics, v.24, n.1, p.69-87, 1993.

WALTERS, M. B.; FIELD, C. B. Photosynthetic light acclimation in two rainforest Piper species with diferent ecological amplitudes. Oecologia, v.72, n.3, p.449-456, 1987 